\title{
Influence of hydrogen bonds and temperature on dielectric properties
}

\author{
Jordi Ortiz de Urbina and Gemma Sesé*, \\ Departament de Física, \\ Universitat Politècnica de Catalunya, \\ Campus Nord-Mòdul B4, \\ c/ Jordi Girona 1-3, \\ 08034 Barcelona, Spain
}

June 2, 2016

\begin{abstract}
Dielectric properties have been evaluated by means of Molecular Dynamics simulations on two model systems made up by dipolar molecules. One of them mimics methanol, whereas the other differs from the former only in the ability of forming hydrogen bonds. Static dielectric properties such as permittivity and the Kirkwood factor have been evaluated, and results have been analyzed by considering the distribution of relative orientations between molecular dipoles. Dipole moment time correlation functions have also been evaluated. The relevance of contributions associated to autocorrelations of molecular dipoles and to cross-correlations between dipoles belonging to different molecules has been investigated. For methanol, the Debye approximation for the overall dipole moment correlation function is not valid at room temperature. The model applies when hydrogen bonds are suppressed, but it fails upon cooling the non-associated liquid. Important differences between relaxation times associated with dipole auto and cross-correlations as well as their relative relevance are at the root of the Debye model breakdown.
\end{abstract}

*e-mail:gemma.sese@upc.es 


\section{INTRODUCTION}

The search for the microscopic mechanisms governing dielectric response in polar liquids is a topic of fundamental interest. To this end, experimental measurements on slightly different materials can be performed and the role of such differences onto the measured properties can be analyzed. Dielectric spectroscopy has been used, for exemple, to elucidate how the molecular structure of polar compounds influences their response to external electric fields, and also how this response changes at temperatures approaching glass transition [1]. Generally speaking, results emphasize the relevance of intermolecular interactions in governing relaxation dynamics.

Because of their role in many chemical processes, hydrogen bonded (HB) liquids have attracted much attention. Several features characterizing their dielectric behaviour have been attributed to the properties of their corresponding HB networks $[2,3,4,5]$. Among HB liquids, relaxation in monoalcohols has been investigated by using a variety of experimental techniques. It is generally accepted that their dielectric spectra show three processes. Nevertheless, they are material dependent, and the conditions at which the structural and the secondary peak merge, as well as the nature of the third Debye peak are still under debate $[6,7]$. In addition, the study of the microscopic mechanisms governing their macroscopic dielectric properties can help to advance onto the understanding of systems with more complicated HB structures.

Computer simulation is a complementary technique that can be used to unveil the signatures of molecular model details onto macroscopic properties. Large samples and long simulations are required in order to obtain statistically significant results for the dielectric properties, which are collective in nature. For low weight molecular liquids, it was shown that samples of a few hundreds of particles behave like macroscopic dielectric materials $[8,9]$. Simplified molecular models have been considered in computational studies of HB liquids because they allow simulations to span over larger time intervals. Then, united atom models have been used to evaluate dielectric properties on alcohols like methanol [10,11] and ethanol [12] at room temperature by using Molecular Dynamics simulations.

The purpose of our work is twofold. On the one hand, we intend to use simulations to get some insight onto the influence of HB on dielectric properties of a methanol model at room temperature. Both static and dynamic properties will be obtained for methanol and for an ideal methanol-like system whose molecules have the same dipolar moment as those in methanol but that lack sites for hydrogen bonding. It should be noted that $99 \%$ of methanol molecules are hydrogen bonded at room temperature [13]. The role of HB on ionic association [14], single particle dynamics $[15,16,17]$ and thermodynamic properties [18] was previously investigated for this system. On the other hand, the influence of temperature on the dielectric properties 
of the non-associated liquid at very low temperatures will also be studied. It has been previously obtained that systems composed by Lennard-Jones rigid asymmetric diatomic molecules exhibit both structural and secondary relaxation processes in rotation below an onset temperature $[19,20]$. We intend to check on the dielectric relaxation behaviour of a system made up by sligthly asymmetric diatomic molecules, with an added constant dipole moment. Results will also be tested against existing theoretical models.

The paper is organized as follows. In section 2, simulation details and computed correlation functions are presented. Results of static and dynamic dielectric properties are included in section 3, which are also tested against existing theoretical models. Some concluding remarks are gathered in the last section.

\section{MODELS AND METHODOLOGY}

Molecular dynamics simulations of a system that mimicks methanol $(\mathrm{MeOH})$ have been performed. A three-site rigid molecular model has been used. Molecular sites belonging to different molecules interact by means of electrostatic and Lennard-Jones forces according to an OPLS potential [21]. The Ewald summation [22] has been used in the evaluation of electrostatic interactions. A system composed of rigid diatomic neutral molecules has also been simulated $(\mathrm{MeO})$. The model is similar to the one considered for $\mathrm{MeOH}$ but hydrogen sites have been suppressed so that $\mathrm{HB}$ cannot be stablished. Two interacting sites per molecule have been considered and short range Lennard-Jones interactions are coincident with those used for the $\mathrm{MeOH}$ sample. Their masses are the ones of oxygen and of the methyl group respectively. They have been assigned charges such that the molecular dipole moment equals that of methanol molecules (2.22 D) [15] . Potential parameters are gathered in table I.

The simulated samples are made up of $\mathrm{N}=1000$ molecules located in a cubic box with periodic boundary conditions. After being equilibrated at room temperature, the $\mathrm{MeO}$ system has been quenched at constant pressure according to the procedure described in [16]. For ten selected temperatures between $298 \mathrm{~K}$ and $123 \mathrm{~K}$, the samples have been equilibrated. Production runs of $1 \mathrm{~ns}$ at the highest temperature and of $6 \mathrm{~ns}$ at the lowest temperature have been performed in the $(\mathrm{N}, \mathrm{V}, \mathrm{T})$ ensemble. Computed glass transition temperature for $\mathrm{MeO}$ is $95 \mathrm{~K}$ [17].

Correlation functions of the system's dipole density have been obtained in order to study dielectric properties. They involve longitudinal $\left(\vec{M}_{L}(\vec{k}, t)\right)$ and transverse $\left(\vec{M}_{T}(\vec{k}, t)\right)$ Fourier components of the dipole density, which are defined as 


$$
\begin{gathered}
\vec{M}_{L}(\vec{k}, t)=\sum_{j=0}^{N} \hat{k} \hat{k} \cdot \vec{\mu}_{j}(t) \exp \left[i \vec{k} \vec{r}_{j}(t)\right] \\
\vec{M}_{T}(\vec{k}, t)=\sum_{j=0}^{N}(1-\hat{k} \hat{k}) \cdot \vec{\mu}_{j}(t) \exp \left[i \vec{k} \vec{r}_{j}(t)\right],
\end{gathered}
$$

where $\overrightarrow{\mu_{j}}$ is the dipole moment of molecule $j$, and $\overrightarrow{r_{j}}(t)$ is the position of the molecule's center of mass. $\vec{k}$ should be compatible with the box length $L$, specifically, $\vec{k}=2 \pi / L(l, m, n)$, where $L$ is the box length, $l, m$ and $n$ are integers, and $\hat{k}$ is the corresponding unit vector. Their correlations have been evaluated according to

$$
\Phi_{A}(\vec{k}, t)=<\vec{M}_{A}(\vec{k}, t) \cdot \vec{M}_{A}(-\vec{k}, 0)>/<\left|\vec{M}_{A}(\vec{k}, 0)\right|^{2}>,
$$

where $A$ can refer to the longitudinal $(L)$ component or to the transverse (T) component. By means of the linear response theory it is possible to relate the components of the susceptibility tensor $\chi_{A}^{0}(\vec{k}, w)$ to $\Phi_{A}(\vec{k}, t)$ for a system of rigid and non-polarizable molecules through [23]

$$
\chi_{A}^{0}(\vec{k}, w)=\left[1+i w \Phi_{A}(\vec{k}, w)\right]<\left|\vec{M}_{A}(\vec{k}, 0)\right|^{2}>/\left(\nu_{A} L^{3} k_{b} T \varepsilon\right),
$$

being $\nu_{L}=1$ and $\nu_{T}=2$, and

$$
\Phi_{A}(\vec{k}, w)=\int_{0}^{\infty} d t \Phi_{A}(\vec{k}, t) \exp (i w t) .
$$

Then, the longitudinal $\left(\varepsilon_{L}(\vec{k}, w)\right)$ and transverse $\left(\varepsilon_{T}(\vec{k}, w)\right)$ components of the dielectric permittivity tensor are

$$
\begin{aligned}
& \frac{\varepsilon_{L}(\vec{k}, w)-1}{\varepsilon_{L}(\vec{k}, w)}=\chi_{L}^{0}(\vec{k}, w) \\
& \varepsilon_{T}(\vec{k}, w)-1=\chi_{T}^{0}(\vec{k}, w) .
\end{aligned}
$$

For the static case $(w=0)$ and for an isotropic system, it is possible to write, using that $y=\frac{4 \pi}{9} \rho \mu^{2} /\left(k_{b} T\right)$,

$$
\begin{aligned}
& \frac{\varepsilon_{L}(k)-1}{\varepsilon_{L}(k)}=y \frac{<\left|\vec{M}_{L}(k, 0)\right|^{2}>}{N \mu^{2}}, \\
& \varepsilon_{T}(k)-1=y \frac{<\left|\vec{M}_{T}(k, 0)\right|^{2}>}{2 N \mu^{2}} .
\end{aligned}
$$

For a system big enough, that is, when $k$ is sufficiently small,

$$
\lim _{k \rightarrow 0} \varepsilon_{L}(k) \approx \lim _{k \rightarrow 0} \varepsilon_{T}(k) \approx \varepsilon,
$$


being $\varepsilon$ the dielectric permittivity of the system, which can also be evaluated in a system of non-polarizable molecules by using [24]

$$
\epsilon=1+\frac{4}{3} \pi \rho \beta \mu^{2} g
$$

where $\rho$ is the molecular density, $\beta=1 /\left(k_{B} T\right)$ and $\mu$ is the molecular dipole moment modulus. $g$ is the finite system Kirkwood factor, which can be evaluated in simulations by means of $[8,25]$

$$
g=\frac{\left(\sum_{i} \overrightarrow{\mu_{i}}\right)^{2}}{N \mu^{2}}
$$

For a system of orientationally uncorrelated dipoles, $g=1$. If the dipoles tend to be parallel, $g>1$. Otherwise, $g<1$. By using Kirkwood's formula $[26,27]$, the Kirkwood factor for an infinite system can be calculated as

$$
g^{0}=g \frac{(2 \epsilon+1)}{3 \epsilon} .
$$

It is also possible to evaluate the frequency dependent permittivity $(\varepsilon(\omega))$ from the total dipole moment correlation function

$$
\Phi(t)=\frac{\langle\vec{M}(0) \cdot \vec{M}(t)\rangle}{\left\langle|\vec{M}(0)|^{2}\right\rangle},
$$

where $\vec{M}(t)$ is the dipole moment of the sample at time $t$, which is related to the individual molecular dipole moments $\vec{M}(t)=\sum_{i}^{N} \vec{\mu}_{i}(t)$. The real and imaginary components of $\varepsilon(\omega)$ are respectively [28]

$$
\begin{gathered}
\varepsilon^{\prime}(w)=\varepsilon-(\varepsilon-1) \omega \int_{0}^{\infty} \Phi(t) \sin (w t) d t \\
\varepsilon^{\prime \prime}(w)=(\varepsilon-1) \omega \int_{0}^{\infty} \Phi(t) \cos (w t) d t
\end{gathered}
$$

\section{RESULTS}

\subsection{STATIC DIELECTRIC PROPERTIES}

The finite system Kirkwood factor has been evaluated in our simulations by means of equation (12). Its values are gathered in table II, as well as the ones for the permittivity and the infinite system Kirwook factor, which have been obtained by using equations (11) and (13) respectively. The evaluated permittivity for $\mathrm{MeOH}$ is 20 , which is significantly lower than the experimental value obtained for methanol (32) [29]. This is the consequence of the approximations of the model such as the neglect of polarizability and the use of united-atom sites. It reasonably agrees with previous works [10] 
that considered the same approximations with slightly different potential parameters.

It is apparent from results in table II that suppressing HB results in smaller values for the Kirkwood factors and, consequently, for the permittivity. They are about $25 \%$ lower than the ones obtained for $\mathrm{MeOH}$. This is consistent with the fact that, even though the molecular dipole is the same for both systems, the proportion of electrostatic energy is larger in the system with HB [30]. In the latter, electrostatic interactions are highly directional, whereas they tend to be isotropic in the non-associated system.

Upon cooling, the Kirkwood factors and the permittivity of $\mathrm{MeO}$ increase. Taking into account that the modulus of the molecular dipole moment is constant, this behaviour should be related to an increase of correlations between the orientation of dipole moments belonging to different molecules, as will be subsequently discussed.

The function $\langle\cos (\theta(r))\rangle$, being $\theta$ the angle between the dipole moments of two molecules whose centers of mass are separated a distance $r$, is displayed in the inset of figure 1. At room temperature, it is apparent that orientational correlations between dipole moments belonging to different molecules rapidly decrease with distance, and that the contribution of the first shell is the most important in both systems. It is also shown that $\mathrm{MeOH}$ molecules located within the first shell of a given one tend to align their molecular moments. This restricted orientation between dipoles is retained at larger distances, which is consistent with a linear hydrogen-bonded network. This is not true for the non-associated system, where orientation tends to be antiparallel for the closest molecules, but parallel orientation is also possible within the first shell. In this case, Kirwood factors result from the addition of positive (parallel) and negative (antiparallel) terms, which leads to smaller values than those obtained for methanol.

The behaviour of $\langle\cos (\theta(r))\rangle$ for the non-associated system doesn't qualitatively change upon cooling, as it occurs for most pair distribution functions [30]. It is apparent in figure 1, though, that orientational correlations tend to increase not only between molecules within the first coordination shell. Moreover, the relevance of correlations with molecules in the second shell increases significantly at low temperatures.

The dependence of the Kirkwood factor with $r$ has been analyzed by means of $[8]$

$$
g_{\kappa}(r)=1+4 \pi \rho \int_{0}^{r} g_{c m}(r)<\cos (\theta(r))>r^{2} d r,
$$

where $g_{c m}(r)$ is the radial distribution function for molecular centers-ofmass. $g_{\kappa}(r)$ functions are displayed in figure 1 . At room temperature, the results for $\mathrm{MeOH}$ are qualitatively different from the ones of $\mathrm{MeO}$. Whereas restrictions in the orientation of $\mathrm{MeOH}$ molecules prevent the function from oscillate as the integral is extended to larger distances, marked oscillations 
are apparent in the non-associated system. These oscillations become more important upon cooling the system. In addition, it is apparent that the contribution of dipoles located in the second solvation shell to $g_{\kappa}(r)$ experiences the largest increase as temperature decreases.

Values for the longitudinal and transverse components of the wave-vector static permittivity constant, defined in eqs. (8) and (9) respectively, are gathered in table III. For all systems, eq (10) is fulfilled for the transverse component, which proves that the simulated samples are big enough so that the results can be considered as representative of a macroscopic isotropic fluid. Discrepancies observed for the longitudinal component can be attributed to the fact that, according to eq. (8), $\varepsilon_{L}(k)$ displays a singularity for $y=\left(N \mu^{2}\right) /\left(\left|\vec{M}_{L}(k, 0)\right|^{2}\right)$, which is numerically very close to the $y$ values obtained for our systems [10].

\subsection{DYNAMIC DIELECTRIC PROPERTIES}

The total dipole moment correlation function $\Phi(t)$, defined in (14), can be decomposed into self and distinct contributions according to

$$
\Phi(t)=\frac{N \mu^{2}}{\left\langle|\vec{M}(0)|^{2}\right\rangle} \Phi_{s}(t)+\frac{N(N-1)\left\langle\vec{\mu}_{i}(0) \cdot \vec{\mu}_{j}(0)\right\rangle}{\left\langle|\vec{M}(0)|^{2}\right\rangle} \Phi_{d}(t),
$$

where $\Phi_{s}(t)$ is the self-correlation function of individual dipoles and $\Phi_{d}(t)$ is the cross-correlation function between dipoles corresponding to different molecules

$$
\begin{gathered}
\Phi_{s}(t)=\frac{\left\langle\vec{\mu}_{i}(t) \cdot \vec{\mu}_{i}(0)\right\rangle}{\left\langle\left|\vec{\mu}_{i}(0)\right|^{2}\right\rangle}, \\
\Phi_{d}(t)=\frac{\left\langle\vec{\mu}_{i}(t) \cdot \vec{\mu}_{j}(0)\right\rangle}{\left\langle\vec{\mu}_{i}(0) \cdot \vec{\mu}_{j}(0)\right\rangle},
\end{gathered}
$$

whith $\mathrm{i} \neq \mathrm{j}$. The finite system Kirkwood factor defined in eq. (12) can be also evaluated as

$$
g=1+(N-1)\left\langle\vec{\mu}_{i}(0) \cdot \vec{\mu}_{j}(0)\right\rangle / \mu^{2},
$$

which leads to the following more compact expression for $\Phi(t)$

$$
\Phi(t)=\frac{1}{g} \Phi_{s}(t)+\left(1-\frac{1}{g}\right) \Phi_{d}(t) .
$$

Results for $\Phi(t)$ at room temperature have been displayed in figure 2 . On a short time scale, some oscillations appear in $\mathrm{MeOH}$ but not in the nonassociated system. A similar behaviour is apparent in $\Phi_{s}(t)$. They are probably a signature of the librational dynamics associated with the existence of $\mathrm{HB}$, as suggested in previous simulations of ethanol [12]. The long time decay of both functions cannot be properly modelled by exponentials, and they 
are best fit with stretched exponential functions $\left(\Phi_{s}(t)=A \exp \left(-\left(t / \tau_{1}\right)^{\beta_{1}}\right)\right.$ and $\Phi(t)=A \exp \left(-\left(t / \tau_{\Phi}\right)^{\beta_{\Phi}}\right)$, respectively). Exponent values and relaxation times are gathered in table IV. It is remarkable that $\tau_{\Phi}$ takes the same value as the HB lifetime in methanol [31], which confirms that it is the breaking of an HB that makes possible the orientation of the liberated dipoles, as previously suggested [32]. As shown in table IV, $\tau_{\Phi}>\tau_{1}$ in $\mathrm{MeOH}$, whereas $\tau_{\Phi} \approx \tau_{1}$ when suppressing HB. For the latter system, both $\Phi_{s}(t)$ and $\Phi(t)$ display the same qualitative behaviour: fast initial decays followed by exponential-like long time relaxation periods, much shorter than the ones observed for the HB liquid.

Upon cooling the $\mathrm{MeO}$ system, the exponential long time decay becomes stretched exponential, even though the exponent never takes values shorter than 0.8 at the analyzed temperatures. It is apparent in table IV that relaxation times increase upon cooling and that the ones for $\Phi(t)$ are larger than the ones obtained for $\Phi_{s}(t)$ at all temperatures. Moreover, differences between them increase as temperature decreases, suggesting that cross-correlations become more important.

The Debye model considers a liquid as a viscous continuum in which the dispersed molecules perform isotropic Brownian reorientations. Then, it states that $\Phi(t)$ displays an exponential long-time decay which can be approximated by the single molecule reorientation function $\Phi_{s}(t)$ [33]. From our results, $\mathrm{MeOH}$ appears as a non-Debye fluid at room temperature. Oppositely, $\mathrm{MeO}$ is a Debye fluid at high temperatures, but a breakdown of the Debye model occurs at low temperatures. It is interesting to analyze the relative contribution of cross-correlations to the overall $\Phi(t)$ function. Results are displayed in figure 3. At room temperature, the contribution of crosscorrelations to $\Phi(t)$ for $\mathrm{MeOH}$ is about $60 \%$, whereas in the non-associated system, it is about $45 \%$. For the non-associated system, the Debye model is valid at the higher analyzed temperatures not because cross-correlations are unimportant but because their associated relaxation times are similar to the ones of self-correlations. Upon cooling the system, the relevance of the cross-correlation term increases significantly, up to $70 \%$ at the lowest analyzed temperature, as shown in figure 3 . Then, both the increase of the relative relevance of cross-correlations and the rise of the difference between time scales of self- and cross-correlations are at the root of the Debye model breakdown.

$\Phi_{T}(\vec{k}, t)$ and $\Phi_{L}(\vec{k}, t)$, defined by eq.(3), have also been calculated. The three smallest possible values for $k$ have been considered, and a very mild dependence on $k$ has been obtained over this range. It has been found that the transverse function $\Phi_{T}(\vec{k}, t)$ is very similar to $\Phi(t)$ for all systems. Longitudinal functions $\Phi_{L}(\vec{k}, t)$ are much short-lived in all cases, as shown in figure 4. In addition, a fast oscillatory decay is obtained for $\mathrm{MeOH}$, whereas the initially monotonically decreasing function is followed by pronounced overdamped oscillations in the non-associated system. Then, suppression of 
HB modifies, even qualitatively, the initial decay of $\Phi_{L}(\vec{k}, t)$. Decreasing the temperature does not affect the initial decay of the function, as shown in figure 4 .

Within the theoretical framework of the Debye model, the components of the frequency dependent permittivity, defined by equations (15) and (16), can be approximated by

$$
\begin{gathered}
\varepsilon^{\prime}(w)-1=\frac{\varepsilon-1}{1+w^{2} \tau_{1}^{2}} \\
\varepsilon^{\prime \prime}(w)=\frac{(\varepsilon-1) w \tau_{1}}{1+w^{2} \tau_{1}^{2}} .
\end{gathered}
$$

Recalling that $\varepsilon(w)=\varepsilon^{\prime}(w)-i \varepsilon^{\prime \prime}(w)$, they can be merged into

$$
\frac{\varepsilon(w)-1}{\varepsilon-1}=\frac{1}{1+i w \tau_{1}} .
$$

Davidson-Cole modified eq.(25) by adding a parameter $\beta$ [34]

$$
\frac{\varepsilon(w)-1}{\varepsilon-1}=\frac{1}{(1+i w \tau)^{\beta}}
$$

where $0<\beta \leq 1$. Using that $\tan (\phi)=w \tau$,

$$
\begin{gathered}
\frac{1}{(1+i w \tau)^{\beta}}=\frac{1}{\left(1+i \frac{\sin (\phi)}{\cos (\phi)}\right)^{\beta}}=\left[\cos ^{\beta}(\phi) e^{-i \phi \beta}\right] \\
\varepsilon^{\prime}=\cos ^{\beta}(\phi) \cos (\beta \phi), \\
\varepsilon^{\prime \prime}=\cos ^{\beta}(\phi) \sin (\beta \phi) .
\end{gathered}
$$

Real and imaginary components of the frequency dependent permittivity at room temperature are displayed in figure 5, as well as recent experimental measurements for methanol [29]. In order to leave aside the discrepancies observed for the static permittivity, functions $\left(\varepsilon^{\prime}(w)-1\right) /(\varepsilon-1)$ and $\varepsilon^{\prime \prime}(w) /(\varepsilon-1)$ are plotted. Calculations on $\mathrm{MeOH}$ qualitatively reproduce experimental results. According to them, one main peak is apparent at the imaginary component of the permittivity. Some quantitative discrepancies arise on the time scale of the real component and on the peak frequency of the imaginary component. It is apparent that functions obtained in simulations are shifted towards larger frequencies when compared with experiment. Similar behaviour was observed for a four-site model with rigid bonds of ethanol [12]. The neglect of polarizability is probably at the root of these discrepancies, as previously argued [11]. Additionally, the coarse-grained nature of the molecular model might also be a relevant factor. Fast vibrational motions, suppressed by coarse graining, might hinder molecular relaxation, 
resulting in larger experimental relaxation times. Fits of the simulation results to the Davidson-Cole equations (28) and (29) are also displayed in figure 5 , and a reasonable agreement has been encountered.

Suppression of HB produces shifts of real and imaginary components of the frequency dependent permittivity towards larger frequencies. Upon cooling, both components shift towards lower frequencies, and they intersect at frequencies larger than the one corresponding to the imaginary component peak. It is apparent in figure 5 that the Debye model is a very good approximation for the non-associated system at room temperature. Upon cooling the system, only one primary relaxation process is detected, similarly to the results obtained for slighlty asymmetric Lennard-Jones molecules [20]. The Debye model is less satisfactory at low temperatures, and the Davidson-Cole approach, shown in figure 6 , produces better results. This is consistent with the emerging dynamic heterogeneities in the system [35].

\section{CONCLUDING REMARKS}

Dielectric properties have been computed in two model systems which differ only in the ability of establishing hydrogen bonds. One of them mimicks methanol. It has been obtained that the Kirkwood factor and, consequently, the permittivity decrease significantly when suppressing hydrogen bonds. The Kirkwood factor is larger in methanol as a consequence of more restricted relative orientations between molecular dipoles. Specifically, methanol molecules located within the first shell of a given one tend to align their molecular dipoles, whereas both parallel and antiparallel orientations are allowed in the non-associated system. Upon cooling this liquid, the Kirkwood factor also increases, and the contribution of dipoles located within the second solvation shell experiences the largest increase.

When analyzing the dipole correlation function for the non-associated liquid at ambient temperature, it has been found that the contribution of dipole autocorrelations is slightly more important than the one of correlations between dipoles belonging to different molecules. Conversely, correlations between orientations of different molecules are more relevant in methanol. In addition, relaxation times associated with the self and the distinct parts differ significantly, whereas they are quite similar in the system without hydrogen bonds. Consequently, the Debye model reproduces the long time regime of the non-associated system but not the one of methanol at room temperature. For very low temperatures, relaxation in the nonassociated liquid cannot be reproduced by the Debye model. The Debye breakdown takes place not only because the relative relevance of crosscorrelations increases but also because differences between self- and crosscorrelation time scales increase. In addition, it has been confirmed for methanol that relaxation of the overall dipole correlation function is as- 
sociated with the breaking of hydrogen bonds.

The wave vector dependence of the dipole density time correlation function has also been studied. This function has been splitted into its transverse and its longitudinal parts. The behaviour of the transverse component is very similar to the one of the overall dipole time correlation function. The longitudinal component relaxes considerably faster than the transverse part in both systems. Librations appearing at short times in methanol disappear when hydrogen bonds are removed, and the function displays an overdamped oscillatory behaviour.

\section{Acknowledgements}

Finantial support of MINECO (Project FIS2012-39443-C02-01) is acknowledged. 


\section{References}

[1] Z. Chen, D. Bi, R. Liu Y. Tian, L.-M. Wang and K.L. Ngai, Chem. Phys. Lett. 551, 81 (2012).

[2] L.-M. Wang and R. Richert, J. Chem. Phys. 121, 11170 (2004).

[3] B.D. Watode, P.G. Hudge and A.C. Kumbharkhane, J. Mol. Liq. 198, 51 (2014).

[4] V. Conti Nibali and M. Havenith, J. Am. Chem. Soc. 136, 12800 (2014).

[5] H.A. Chaube, V.A. Rana, P. Hudge and A.C. Kumbharkhane, J. Mol. Liq. 193, 29 (2014).

[6] D. Fragiadakis, C. M. Roland and R. Casalini, J. Chem. Phys. 132, 144505 (2010).

[7] R.Böhmer, C. Gainaru and R. Richert, Physics Reports 545, 125 (2014).

[8] M. Neumann, O. Steinhauser and G.S. Pawley, Molec. Phys. 52, 97 (1984).

[9] D.M.F. Edwards, P.A. Madden and I.R. McDonald, Molec. Phys. 51, 1141 (1984).

[10] T. Fonseca and B.M. Ladanyi, J. Chem. Phys. 93, 8148 (1990).

[11] M.S. Skaf, T. Fonseca and B.M. Ladanyi, J. Chem. Phys. 98, 8929 (1993).

[12] L. Saiz, E. Guàrdia and J. A. Padró, J. Chem. Phys. 113, 2814 (2000).

[13] R. Palomar and G. Sesé, J. Chem. Phys. 133, 044501 (2010).

[14] F. Hirata and R. M. Levy, J. Phys. Chem. 91, 4788 (1987).

[15] E. Guàrdia, G. Sesé and J. A. Padró, J. Mol. Liq. 62, 1 (1994).

[16] R. Palomar and G. Sesé, J. Phys. Chem. B 109, 499 (2005).

[17] G. Sesé, J. Ortiz de Urbina and R. Palomar, J. Chem. Phys. 137, 114502 (2012).

[18] D. Fragiadakis and C. M. Roland, J. Chem. Phys. 138, 12 A502 (2013).

[19] D. Fragiadakis and C. M. Roland, Phys. Rev. E 89, 052304 (2014).

[20] D. Fragiadakis and C. M. Roland, Phys. Rev. E 91, 022310 (2015). 
[21] W. L. Jorgensen, J. Phys. Chem. 90, 1276 (1986).

[22] M.P. Allen and D.J. Tildesley. Computer Simulations of liquids (Clarendon Press, Oxford, 1987).

[23] P.A. Madden and D. Kivelson, Adv. Chem. Phys. 56, 467 (1984).

[24] D. Frenkel and B. Smit. Understanding Molecular Simulation. From Algorithms to Applications (Academic Press, San Diego, 2002).

[25] M. Neumann, J. Chem. Phys. 82, 5663 (1985).

[26] J.G. Kirkwood, J. Chem. Phys. 7, 911 (1939).

[27] S.W. de Leeuw, J.W. Perram and E.H. Smith, Annu. Rev. Phys. Chem. 37, 245 (1986).

[28] D. Levesque, J.-J. Weis and D. Oxtoby, J. Chem. Phys. 79, 917 (1983).

[29] H.A. Chaube, V.A. Rana, P. Hudge and A.C. Kumbharkhane, J. Mol. Liq. 211, 346 (2015).

[30] R.Palomar, Ph.D. Thesis, Technical University of Catalonia, 2007. (http://www.tdx.cat/handle/10803/6589)

[31] J. A. Padró, L. Saiz and E. Guàrdia, J. Mol. Sruct. 416, 243 (1997).

[32] C. Brot and M. Magat, J. Chem. Phys. 39, 841 (1963).

[33] D. A. McQuarrie. Statistical Mechanics (University Science Books, California, 2000).

[34] D.W. Davidson and R.H. Cole, J. Chem. Phys. 18, 1417 (1950).

[35] R. Palomar and G. Sesé, Phys. Rev. E 75, 011505 (2007). 


\begin{tabular}{ccccc}
\hline \hline Site & $\sigma(\AA)$ & $\epsilon(\mathrm{Kcal} / \mathrm{mol})$ & $\mathrm{q}(\mathrm{e}) \mathrm{MeOH}$ & $\mathrm{q}(\mathrm{e}) \mathrm{MeO}$ \\
\hline $\mathrm{O}$ & 3.071 & 0.170 & -0.700 & -0.323 \\
$\mathrm{H}$ & 0.0 & 0.0 & 0.435 & \\
$\mathrm{Me}$ & 3.775 & 0.207 & 0.265 & 0.323 \\
\hline \hline
\end{tabular}

Table I: Parameters for the Lennard-Jones potentials used in MD simulations of $\mathrm{MeOH}$ and $\mathrm{MeO}$. Also displayed, electric charges for the $\mathrm{MeOH}$ and for the $\mathrm{MeO}$ sites. 


\begin{tabular}{ccccc}
\hline \hline & $T(\mathrm{~K})$ & $g \pm \sigma_{g}$ & $\epsilon \pm \sigma_{\epsilon}$ & $g^{0} \pm \sigma_{g^{0}}$ \\
\hline $\mathrm{MeOH}$ & 298 & $2.5 \pm 0.2$ & $20 \pm 2$ & $1.7 \pm 0.2$ \\
\hline $\mathrm{MeO}$ & 298 & $1.9 \pm 0.1$ & $15 \pm 1$ & $1.3 \pm 0.1$ \\
& 268 & $2.0 \pm 0.1$ & $16 \pm 1$ & $1.6 \pm 0.1$ \\
& 238 & $2.2 \pm 0.1$ & $23 \pm 1$ & $1.6 \pm 0.1$ \\
& 218 & $2.3 \pm 0.1$ & $25 \pm 1$ & $1.6 \pm 0.1$ \\
208 & $2.5 \pm 0.1$ & $30 \pm 1$ & $1.6 \pm 0.1$ \\
198 & $2.5 \pm 0.1$ & $33 \pm 1$ & $1.7 \pm 0.1$ \\
178 & $2.6 \pm 0.1$ & $39 \pm 1$ & $1.8 \pm 0.1$ \\
158 & $3.1 \pm 0.1$ & $51 \pm 1$ & $2.0 \pm 0.1$ \\
138 & $3.9 \pm 0.1$ & $74 \pm 1$ & $2.0 \pm 0.1$ \\
123 & $3.4 \pm 0.1$ & $75 \pm 2$ & $2.3 \pm 0.2$ \\
\hline \hline
\end{tabular}

Table II: Kirkwood factor $(g)$ and dielectric permittivity $(\epsilon)$ evaluated by using equations (12) and (11) respectively, from simulations of methanol $(\mathrm{MeOH})$ and metoxi $(\mathrm{MeO})$ at several temperatures. Kirkwood factor values corrected according to equation (13) are also listed $\left(g^{0}\right)$. Uncertainties have been estimated from statistical fluctuations. 


\begin{tabular}{cccccc}
\hline \hline$\left(k / k_{\text {min }}\right)^{2}$ & $k / \AA^{-1}$ & $\left\langle\left|\vec{M}_{L}(\vec{k}, 0)\right|^{2}\right\rangle / N \mu^{2}$ & $\left\langle\left|\vec{M}_{T}(\vec{k}, 0)\right|^{2}\right\rangle / 2 N \mu^{2}$ & $\varepsilon_{L}(k)$ & $\varepsilon_{T}(k)$ \\
\hline 1 & 0.1542 & $0.0217 \pm 0.001$ & $0.95 \pm 0.21$ & $0.468 \pm 0.078$ & $20.22 \pm 4.63$ \\
2 & 0.2180 & $0.0212 \pm 0.001$ & $0.92 \pm 0.16$ & $0.473 \pm 0.063$ & $19.71 \pm 3.40$ \\
3 & 0.2671 & $0.0209 \pm 0.001$ & $0.88 \pm 0.14$ & $0.482 \pm 0.061$ & $19.6 \pm 2.88$ \\
\hline \hline 1 & 0.1542 & $0.0215 \pm 0.0004$ & $0.64 \pm 0.04$ & $0.477 \pm 0.032$ & $15.07 \pm 0.857$ \\
2 & 0.2180 & $0.0218 \pm 0.0002$ & $0.63 \pm 0.02$ & $0.484 \pm 0.020$ & $13.81 \pm 0.418$ \\
3 & 0.2671 & $0.0223 \pm 0.0002$ & $0.62 \pm 0.02$ & $0.502 \pm 0.021$ & $12.87 \pm 0.344$ \\
\hline \hline 1 & 0.1635 & $0.0080 \pm 0.0002$ & $1.26 \pm 0.25$ & $0.511 \pm 0.041$ & $82.3 \pm 16.3$ \\
2 & 0.2313 & $0.0081 \pm 0.0002$ & $1.28 \pm 0.11$ & $0.484 \pm 0.043$ & $81.6 \pm 6.9$ \\
3 & 0.2833 & $0.0082 \pm 0.0002$ & $1.13 \pm 0.13$ & $0.502 \pm 0.042$ & $77.1 \pm 8.41$ \\
\hline \hline
\end{tabular}

Table III: Longitudinal and transversal components of the wave-vector dependent static constant for methanol (up) and metoxi at $298 \mathrm{~K}$ (middle) and at $123 \mathrm{~K}$ (down). 


\begin{tabular}{cccccc}
\hline \hline & $T(\mathrm{~K})$ & $\tau_{1} \pm \sigma_{\tau_{1}}$ & $\beta_{1}$ & $\tau_{\phi} \pm \sigma_{\tau_{\phi}}$ & $\beta_{\phi}$ \\
\hline $\mathrm{MeOH}$ & 298 & $11.8 \pm 0.3$ & 0.85 & $16 \pm 10$ & 0.9 \\
\hline \multirow{4}{*}{$\mathrm{CeO}$} & 298 & $0.62 \pm 0.02$ & 1 & $0.64 \pm 0.10$ & 1 \\
& 268 & $0.80 \pm 0.04$ & 1 & $0.86 \pm 0.02$ & 1 \\
& 238 & $1.10 \pm 0.02$ & 1 & $1.14 \pm 0.12$ & 1 \\
& 218 & $1.14 \pm 0.03$ & 0.9 & $1.51 \pm 0.30$ & 0.9 \\
& 208 & $1.34 \pm 0.04$ & 0.9 & $1.98 \pm 0.20$ & 1 \\
& 198 & $1.60 \pm 0.05$ & 0.9 & $2.16 \pm 0.36$ & 0.85 \\
& 178 & $2.26 \pm 0.03$ & 0.9 & $3.30 \pm 0.26$ & 0.9 \\
& 158 & $3.42 \pm 0.06$ & 0.85 & $5.06 \pm 0.74$ & 0.9 \\
& 138 & $7.08 \pm 0.33$ & 0.85 & $13.2 \pm 3.1$ & 0.8 \\
& 123 & $13.8 \pm 1.3$ & 0.8 & $19.0 \pm 5.8$ & 0.9 \\
\hline \hline
\end{tabular}

Table IV: Parameters associated with stretched exponential fits of the self $\left(\Phi_{s}(t)\right)$ dipole correlation functions $\left(\tau_{1}\right.$ and $\left.\beta_{1}\right)$ and of the total $(\Phi(t))$ dipole correlation functions $\left(\tau_{\phi}\right.$ and $\left.\beta_{\phi}\right)$. Estimated errors for the stretched exponents are $5 \%$. 


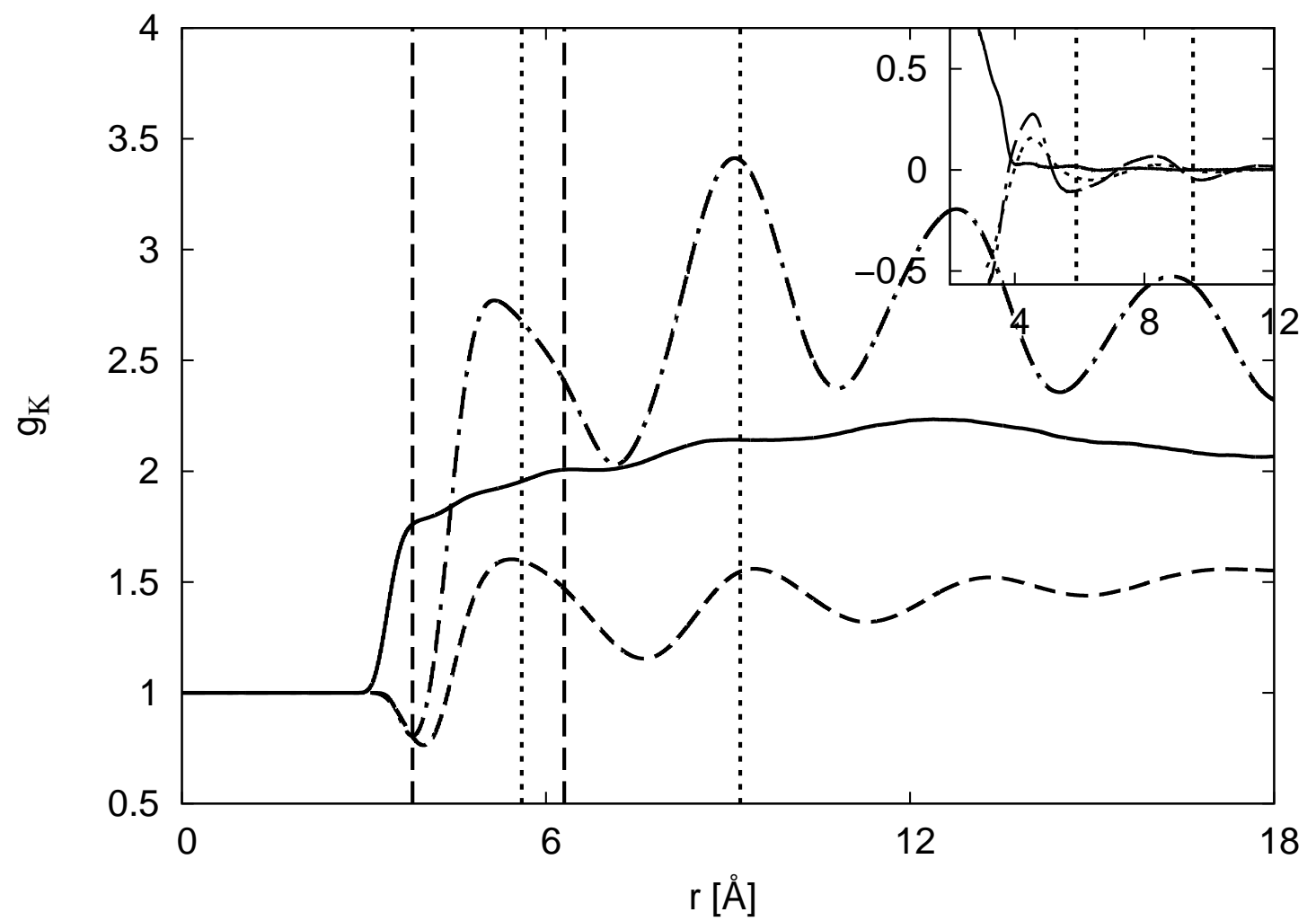

Figure 1: Distance dependent Kirkwood factor $g_{\kappa}(r)$ evaluated according to eq.(17) for $\mathrm{MeOH}$ (continuous line) and $\mathrm{MeO}$ (dashed line) at $298 \mathrm{~K}$, and $\mathrm{MeO}$ at $123 \mathrm{~K}$ (dashed-dotted line). Vertical lines are drawn at limiting distances of the first and second shell of $\mathrm{MeOH}$ (dashed lines) and $\mathrm{MeO}$ (dotted lines). Inset: $\langle\cos (\theta(r))\rangle$ for the same systems, being $\theta$ the angle between dipole moments of two molecules whose centers of mass are separated a distance $r$. Limiting distances of first and second shells for $\mathrm{MeO}$ are also shown (dotted vertical lines). 


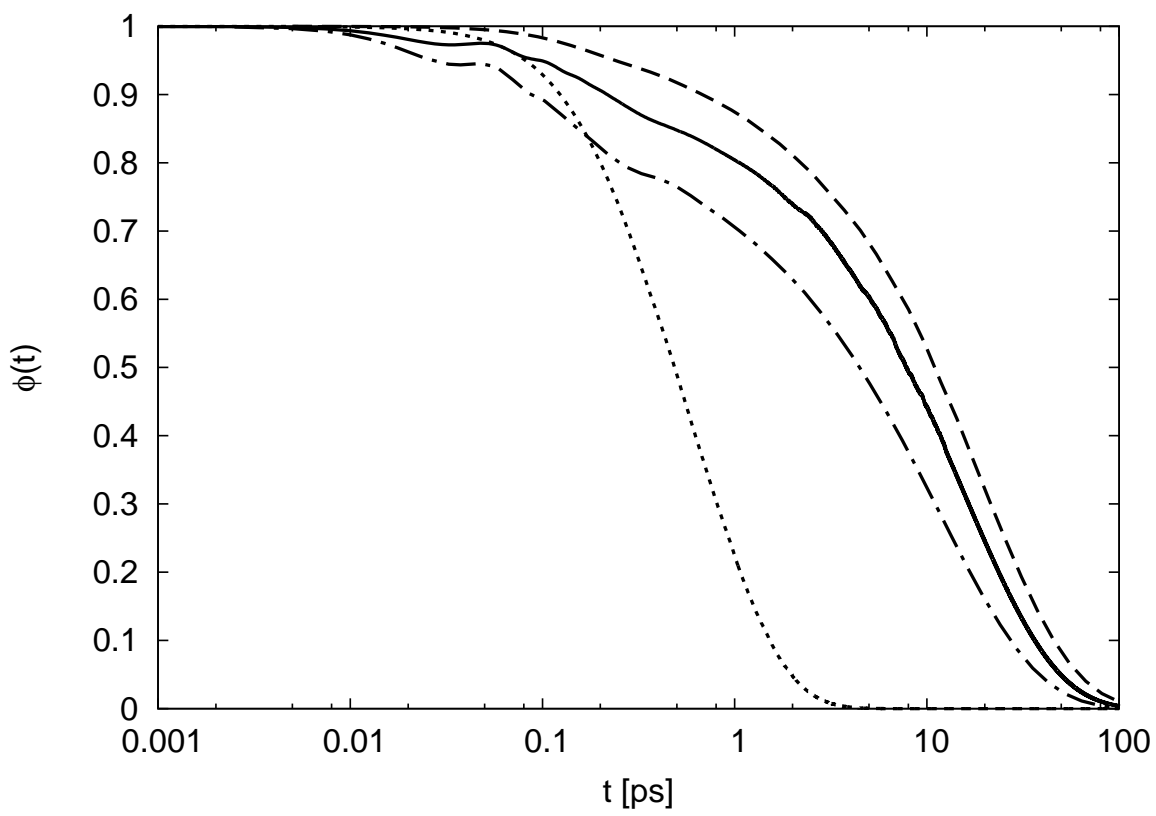

Figure 2: Total dipole moment correlation function $(\Phi(t))$ for $\mathrm{MeOH}$ (continuous line) and $\mathrm{MeO}$ (dotted line) at $298 \mathrm{~K}$, and for $\mathrm{MeO}$ at $123 \mathrm{~K}$ (dashed line). Also shown the self-autocorrelation function of individual dipoles $\left(\Phi_{s}(t)\right)$ for $\mathrm{MeOH}$ at $298 \mathrm{~K}$ (dashed-dotted line). 


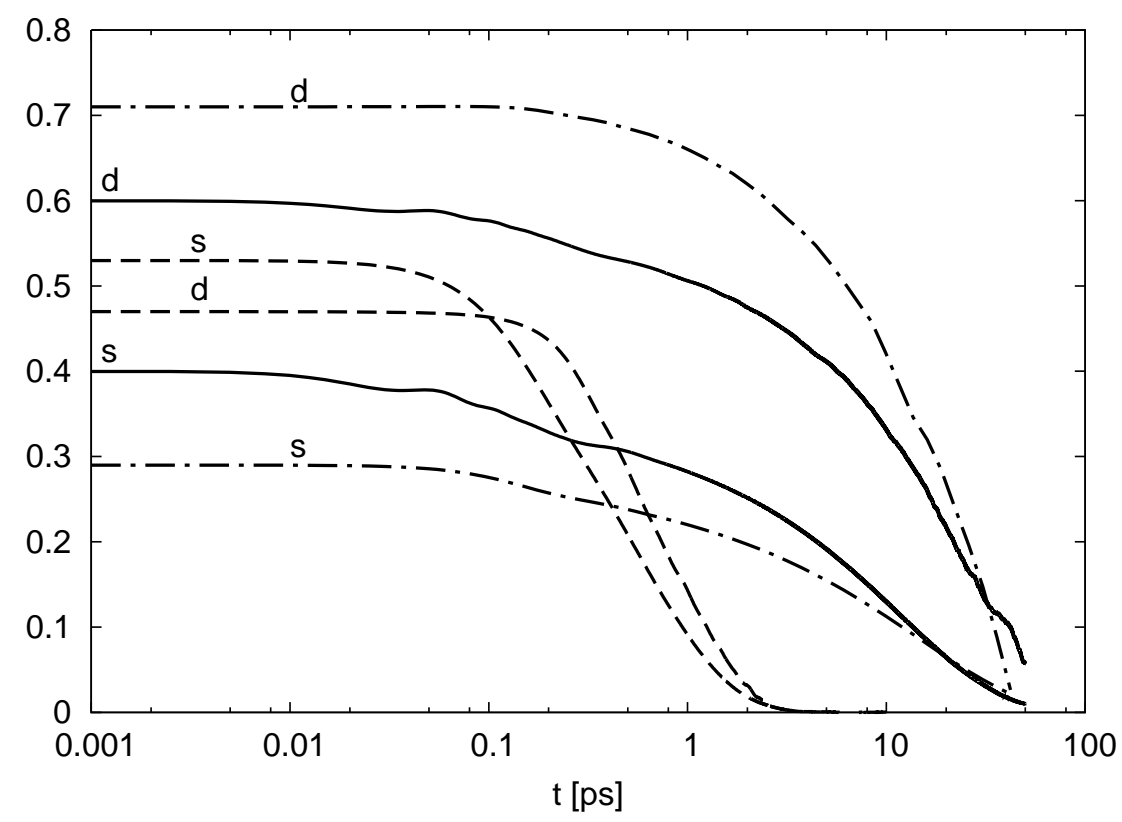

Figure 3: $\operatorname{Self}(\mathrm{s}), \frac{1}{g} \Phi_{s}(t)$, and $\operatorname{distinct}(\mathrm{d}),\left(1-\frac{1}{q}\right) \Phi_{d}(t)$, contributions to the total dipole moment correlation functions for MeOH (continuous lines), and $\mathrm{MeO}$ (dashed lines) at $298 \mathrm{~K}$, and for $\mathrm{MeO}$ at $123 \mathrm{~K}$ (dashed-dotted lines). 


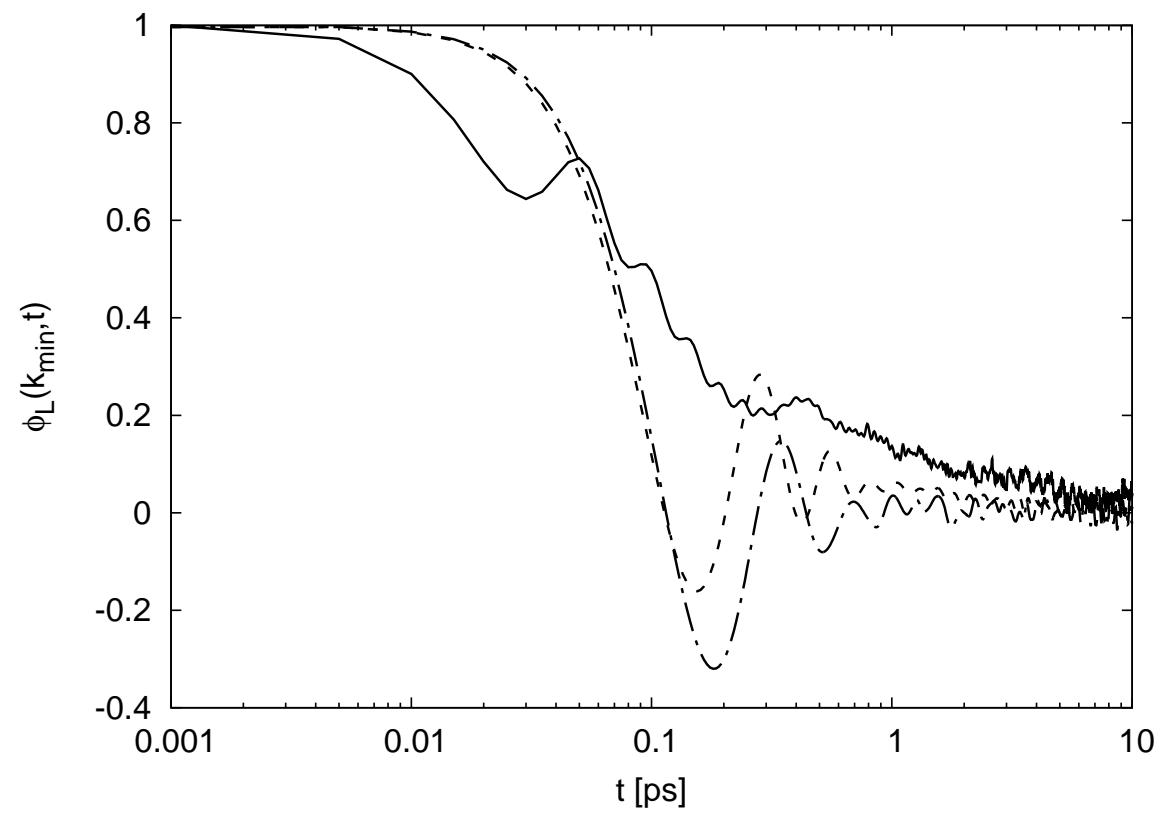

Figure 4: $\Phi_{L}(\vec{k}, t)$ with $k=k_{\text {min }}=2 \pi / L$ for $\mathrm{MeOH}$ (continuous line) and $\mathrm{MeO}$ (dashed-dotted line) at $298 \mathrm{~K}$, and $\mathrm{MeO}$ at $123 \mathrm{~K}$ (dashed line). 


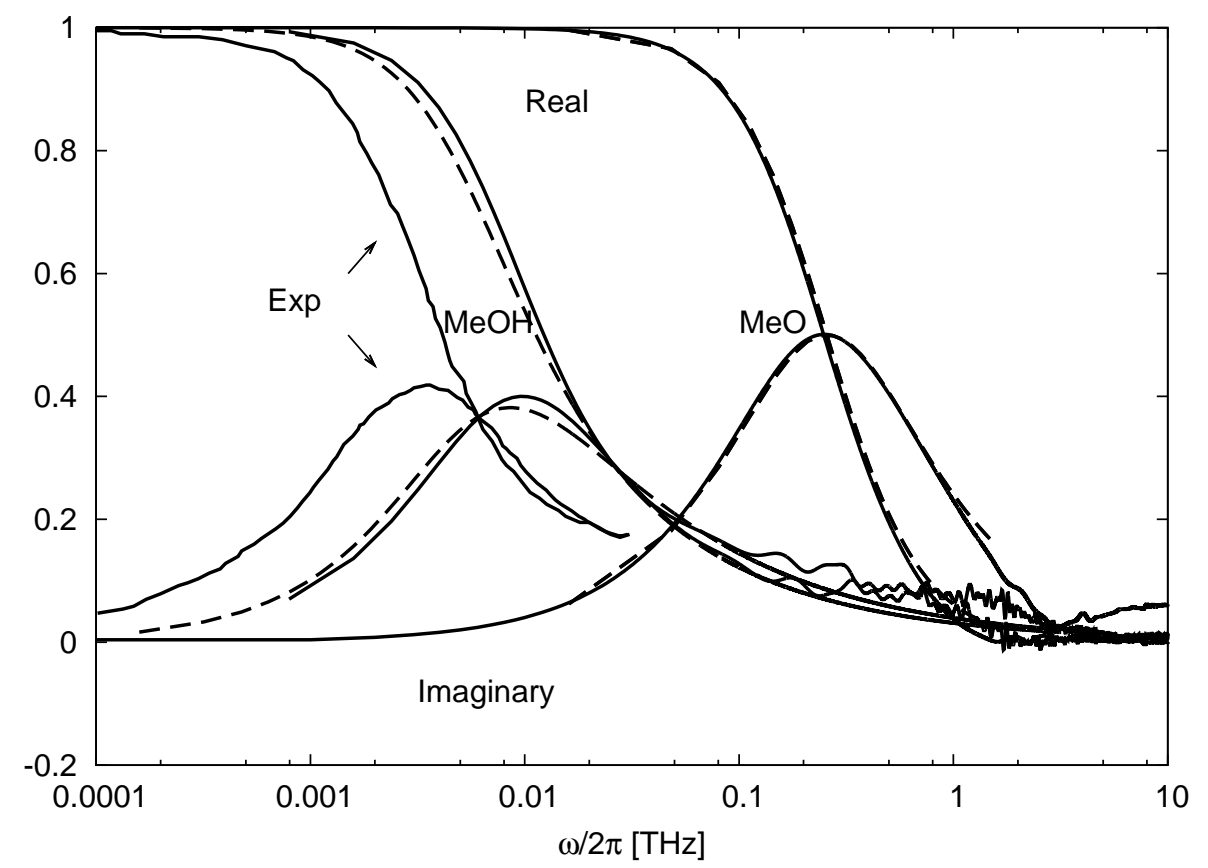

Figure 5: Real $\left(\left(\varepsilon^{\prime}(w)-1\right) /(\varepsilon-1)\right)$ and imaginary $\left(\varepsilon^{\prime \prime}(w) /(\varepsilon-1)\right)$ normalized components of the frequency dependent permittivity for $\mathrm{MeOH}$ and $\mathrm{MeO}$ (continuous lines) at $298 \mathrm{~K}$. Davidson-Cole approach for $\mathrm{MeOH}$ (dashed lines), Debye approximation for $\mathrm{MeO}$ (dashed lines) and experimental (Exp) results for methanol (continuous lines) [29] are also shown. 


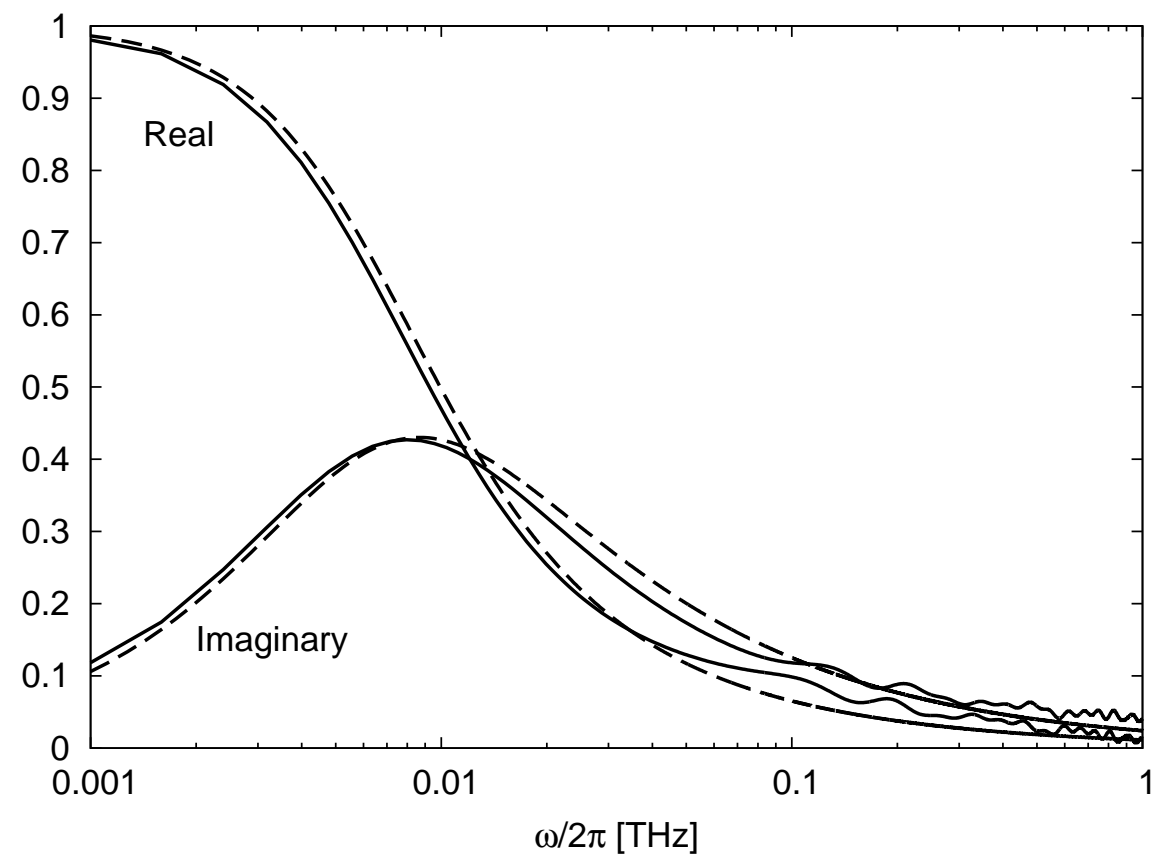

Figure 6: Real $\left(\left(\varepsilon^{\prime}(w)-1\right) /(\varepsilon-1)\right)$ and imaginary $\left(\varepsilon^{\prime \prime}(w) /(\varepsilon-1)\right)$ normalized components of the frequency dependent permittivity for $\mathrm{MeO}$ at $123 \mathrm{~K}$ (continuous lines). Also shown the Davidson-Cole approach (dashed lines). 